\title{
A survey of radiation safety training among South African interventionalists
}

\author{
A Rose, ${ }^{1} \mathrm{MB}$ BCh, MMed (Community Health); W I D Rae, ${ }^{2} \mathrm{MB}$ BCh, PhD \\ ${ }^{1}$ Department of Community Health, Faculty of Health Sciences, University of the Free State, Bloemfontein, South Africa \\ ${ }^{2}$ Department of Medical Physics, Faculty of Health Sciences, University of the Free State, Bloemfontein, South Africa
}

Corresponding author: A Rose (roseas@ufs.ac.za)

Background. Ionising radiation is increasingly being used in modern medicine for diagnostic, interventional and therapeutic purposes. There has been an improvement in technology, resulting in lower doses being emitted. However, an increase in the number of procedures has led to a greater cumulative dose for patients and operators, which places them at increased risk of the effects of ionising radiation. Radiation safety training is key to optimising medical practice.

Objective. To present the perceptions of South African interventionalists on the radiation safety training they received and to offer insights into the importance of developing and promoting such training programmes for all interventionalists.

Methods. In this cross-sectional study, we collected data from interventionalists $(N=108)$ using a structured questionnaire.

Results. All groups indicated that radiation exposure in the workplace is important (97.2\%). Of the participants, the radiologists received the most training (65.7\%). Some participants (44.1\%) thought that their radiation safety training was adequate. Most participants (95.4\%) indicated that radiation safety should be part of their training curriculum. Few (34.3\%) had received instruction on radiation safety when they commenced work. Only $62 \%$ had been trained on how to protect patients from ionising radiation exposure.

Conclusion. Radiation safety training should be formalised in the curriculum of interventionalists' training programmes, as this will assist in stimulating a culture of radiation protection, which in turn will improve patient safety and improve quality of care.

Afr J Health Professions Educ 2018;10(1):10-12. DOI:10.7196/AJHPE.2018.v10i1.981

Interventionalists are highly specialised doctors who undergo rigorous training. The use of ionising radiation is an integral part of their medical practice and potentially poses major occupational health risks, such as skin damage, genetic and chromosomal aberrations, carcinomas and cataract formation. ${ }^{[1]}$ The use of this modality for diagnostic, treatment and interventional procedures has increased substantially, posing greater occupational risks. ${ }^{[2]}$ In medicine, occupational radiation protection is challenging and increased vigilance is required to protect radiation healthcare workers (HCWs). ${ }^{[1]}$

Ionising radiation places patients at risk of developing skin reactions and alopecia, malaise, gastrointestinal problems, damage to heart and lungs, and primary and secondary carcinomas. ${ }^{[3]}$ Patients may receive an increased radiation dose owing to over-investigation, because of the complexity and duration of procedures or poor radiation safety practices by operators. ${ }^{[3]}$ Improved knowledge of radiation safety for patients may assist in reducing these complications and thus improve the quality of care. ${ }^{[4]}$

Specialists require dedicated training in radiation safety, as it effectively reduces radiation risk and optimises radiation safety practices. ${ }^{[5]}$ There is a need to elevate the level of training received by interventional cardiologists to that of interventional radiologists. ${ }^{[6]}$ This may be challenging, as the cardiologists' curriculum already comprises an enormous volume of work, but it is important that professional and regulatory bodies find a way to implement and foster these changes in the interest of interventionalists and their patients. ${ }^{[6]}$
Developing a culture of learning will assist in developing a culture of radiation protection (CRP), which is essential to lessen radiation exposure. A CRP is a combination of the knowledge, beliefs and practices in an organisation that promotes radiation safety in the workplace. ${ }^{[7]}$ Creating and sustaining a CRP is the responsibility of the catheterisation laboratory team (doctors, nurses and radiographers) and managers. ${ }^{[8]}$ The latter are responsible for ensuring that the equipment is functional and maintained and for providing sufficient and correct personal protective equipment (PPE) ${ }^{[8]} \mathrm{A}$ CRP creates awareness of the risks of radiation injury to patients and operators and facilitates improved compliance with PPE use. ${ }^{[7]}$ This culture can be stimulated by including radiation safety training in the formal curriculum of all interventionalists. ${ }^{[9]}$

The objective of this article is to present the findings of the perceptions of South African (SA) interventionalists on the radiation safety training they received and to offer insights into the importance of developing and promoting such training programmes for all interventionalists in SA.

\section{Methods}

In this cross-sectional study, we collected data by means of a structured survey. The study forms part of a larger multiple-methods study, which is described elsewhere. ${ }^{[10]}$

The study population consisted of SA radiologists, adult cardiologists and paediatric cardiologists. Data were collected at cardiology and radiology conferences between May 2015 and September 2016 by an electronic survey 
system (EvaSys, UK) (www.evasys.co.uk) and hard copy. The hyperlink to the survey was emailed to delegates at the conferences and workshops and to academic departments in SA. Hard copies of the survey were handed out at the scientific meetings. There was no randomisation and all eligible interventionalists willing to participate were included in the study. The data were captured electronically, exported to Stata version 14 (StataCorp., USA), and a descriptive analysis was done.

\section{Ethical approval}

Ethical approval was granted by the Human Research Ethics Committee of the Faculty of Health Sciences, University of the Free State, Bloemfontein, SA (ref. no. ECUFS 44/2015). Participants provided written informed consent, and consent was assumed if participants proceeded with the online survey.

\section{Results}

A total of 108 interventionalists completed the survey. Table 1 presents a descriptive analysis of this group, which illustrates their demographic characteristics and the radiation safety training they received.

\section{Discussion}

Interventional procedures place patients and operating staff in the catheterisation laboratory at increased risk of adverse health effects owing to radiation exposure. ${ }^{[1,3]}$ Most participants (97.2\%) ranked occupational radiation exposure as an important consideration (Table 1), which suggests that they were aware that ionising radiation is an occupational risk. It is, however, important to explore their understanding of the risk and its sequelae. Despite technological improvements, resulting in equipment emitting lower doses, low-dose radiation may still have detrimental effects on health. ${ }^{[3]}$ Therefore, training in radiation safety is imperative and essential for protecting staff in the radiation workplace. ${ }^{[11]}$

Overall, participants reported receiving low levels $(35.2 \%)$ of training in radiation safety. Radiologists reported higher levels (65.7\%) of training than cardiologists. These results are similar to those of other studies, where radiologists demonstrated higher levels of knowledge of radiation safety. ${ }^{[9]}$ The median duration of time worked for all participants was 10 (interquartile range 5 - 20) years; participants might therefore have had difficulty recalling their training, which might have introduced bias.

Even though radiobiology and radiation physics are included in the Part I examination for the Fellowship of the College of Diagnostic Radiologists of South Africa, not all the radiologists reported having received training in radiation safety. ${ }^{[12]}$ It is unclear why, despite their training for the Part I examination, radiologists did not report having received training in radiation safety.

It is concerning that there is a difference in training between radiologists and cardiologists, as the interventional procedures performed by these two groups result in similar radiation exposure - placing them at similar

Table 1. Radiation-safety training among South African interventionalists

\begin{tabular}{|c|c|c|c|c|}
\hline Demographic characteristics & $\begin{array}{l}\text { Radiologists, } \\
n=35\end{array}$ & $\begin{array}{l}\text { Adult cardiologists, } \\
n=41\end{array}$ & $\begin{array}{l}\text { Paediatric cardiologists, } \\
n=32\end{array}$ & $\begin{array}{l}\text { Total, } \\
N=108\end{array}$ \\
\hline \multicolumn{5}{|l|}{ Age, years } \\
\hline Median & 43 & 48 & 43 & 44 \\
\hline IQR & $36-49$ & $41-59$ & $39-53$ & $39-53$ \\
\hline Range & $30-60$ & $31-69$ & $32-59$ & $31-69$ \\
\hline \multicolumn{5}{|l|}{ Sex, $n(\%)$} \\
\hline Male & $17(48.6)$ & $37(90.2)$ & $20(62.5)$ & $74(68.5)$ \\
\hline Female & $18(51.4)$ & $4(9.8)$ & $12(37.5)$ & $34(31.5)$ \\
\hline \multicolumn{5}{|l|}{ Worked, years } \\
\hline Median & 11 & 11 & 9 & 10 \\
\hline IQR & $5-16$ & $5-21$ & $5-14$ & $5-20$ \\
\hline Range & $2-32$ & $1-40$ & $1-28$ & $1-40$ \\
\hline \multicolumn{5}{|l|}{ Sector, $n(\%)$} \\
\hline Public & $14(40.0)$ & $11(26.8)$ & $22(68.7)$ & $47(43.5)$ \\
\hline Private & $15(42.9)$ & $23(56.1)$ & $2(6.3)$ & $40(37.1)$ \\
\hline Both & $6(17.1)$ & $7(17.1)$ & $8(25.0)$ & $21(19.4)$ \\
\hline \multicolumn{5}{|l|}{ Perception of occupational radiation exposure, $n(\%)$} \\
\hline Important & $35(100)$ & $39(95.1)$ & $31(96.9)$ & $105(97.2)$ \\
\hline Somewhat important & 0 & $2(4.9)$ & $1(3.1)$ & $3(2.8)$ \\
\hline Received radiation safety training, $n(\%)$ & $23(65.7)$ & $10(24.4)$ & $5(15.6)$ & $38(35.2)$ \\
\hline Training should be part of the curriculum, $n(\%)$ & $34(97.1)$ & $39(95.1)$ & $30(93.8)$ & $103(95.4)$ \\
\hline Received radiation safety induction on commencing work, $n(\%)$ & $19(54.3)$ & $14(34.2)$ & $4(12.5)$ & $37(34.3)$ \\
\hline Received at least one talk on radiation safety, $n(\%)$ & $21(60.0)$ & $25(60.9)$ & $7(21.9)$ & $53(49.1)$ \\
\hline Trained on how to protect patients from radiation, $n(\%)$ & $28(80.0)$ & $25(60.9)$ & $14(43.8)$ & $67(62.0)$ \\
\hline \multirow[t]{2}{*}{ Trained on how to use X-ray equipment, $n(\%)$} & $24(68.6)$ & $20(48.8)$ & $8(25.0)$ & $52(48.2)$ \\
\hline & $n=31$ & $n=24$ & $n=13$ & $N=68$ \\
\hline Considered training adequate, $n(\%)$ & $19(61.3)$ & $8(33.3)$ & $3(23.1)$ & $30(44.1)$ \\
\hline
\end{tabular}


risk. ${ }^{[6]}$ It is important that different specialties employing radiation receive dedicated instruction and training in radiation safety to optimise their medical practice. ${ }^{[13]}$

Most participants (95.4\%) indicated that it was necessary to include radiation safety in the curriculum. Overall, participants indicated low levels of satisfaction (44.1\%) with the level of radiation safety training they had received. The combination of these two factors should encourage the curriculum developers for these two groups to investigate and address this omission, especially for cardiology training. ${ }^{[13]}$

One study indicated that implementation of a training programme resulted in a significant short- and long-term reduction in radiation dose to patients and radiation HCWs. ${ }^{[14]}$ Advocating small behavioural changes among interventionalists reduces radiation during procedures, but requires educating them, especially cardiologists. ${ }^{[15]}$ Encouraging more optimal radiation practices is very difficult and necessitates proactive training strategies. ${ }^{[16]}$ Training in radiation safety greatly improves reduction in radiation dose to patients and operators. ${ }^{[16]}$ Training programmes, however, cannot be a once-off event. In a study by Georges et al. ${ }^{[16]}$ it was found that the duration of the impact of training was up to a maximum of 3 months and then tended to decrease. ${ }^{[16]}$ This suggests that there needs to be continuing reinforcement and training in this field. We suggest that the topic should be part of continuing medical education programmes and incorporated into radiology and cardiology conferences.

Training of interventionalists in radiation safety may have two very important consequences. Firstly, it may increase awareness of ionising radiation as an unseen occupational hazard and facilitate utilisation of PPE to mitigate the effects of radiation. This protects an already scarce and highly skilled healthcare workforce. Secondly, radiation HCWs may become more vigilant when considering the dose administered, thus protecting the patient. Patient safety is the keystone of quality care. ${ }^{[4]}$

\section{Study limitations}

This study did not explore participants' understanding of specific health risks related to ionising radiation. It also did not investigate the participants' thoughts with regard to the content and depth of a radiation safety curriculum. There may be recall bias from participants in reporting the training they received. It should be investigated why all the radiologists did not report having received training in radiation safety. A culture of radiation protection is discussed in an article linked to this study. ${ }^{[17]}$

\section{Conclusion}

Establishing and maintaining an adequate radiation safety training programme is crucial to instilling and sustaining a culture of radiation protection, which can protect radiation workers and patients and improve the quality of care. Radiation safety training should be part of formal training programmes and its importance emphasised for it to be effective. Further research is necessary to determine the areas of deficit in radiation safety among interventionalists and how these can be addressed.

\section{Acknowledgements. None.}

Author contributions. AR conceptualised the study, developed the protocol, collected and analysed the data, and wrote the first and final draft of the manuscript. WIDR conceptualised the study and contributed to the final draft of the manuscript.

Funding. The PhD from which this study emanated was funded by the South African Medical Research Council (SAMRC) under the SAMRC Clinician Researcher Programme. AR received the Discovery Foundation Scholarship, which funded the data collection of this project. SA Heart (Free State Branch) partially funded data collection for the project. WIDR is a recipient of a National Research Foundation incentive grant.

Conflicts of interest. None.

\footnotetext{
1. Smilowitz NR, Balter S, Weisz G. Occupational hazards of interventional cardiology. Cardiovasc Revasc Med 2013;14(4):223-228. https://doi.org/10.1016/j.carrev.2013.05.002

2. Le Heron J, Padovani R, Smith I, Czarwinski R. Radiation protection of medical staff. Eur J Radiol 2010;76(1):2023. https://doi.org/10.1016/.ejrad.2010.06.034

3. Stewart F, Akleyev A, Hauer-Jensen M, et al. ICRP statement on tissue reactions and early and late effects of radiation in normal tissues and organs - threshold doses for tissue reactions in a radiation protection context. Ann ICRP 2012;41(1-2):1-322. https://doi.org/10.1016/j.icrp.2012.02.001

4. Mitchell PH. Defining patient safety and quality care. In: Hughes RG, ed. Patient Safety and Quality: An Evidence Based Handbook for Nurses. Rockville, MD: Agency for Healthcare Research and Quality (US), 2008.

5. Sheyn DD, Racadio JM, Ying J, Patel MN, Racadio JM, Johnson ND. Efficacy of a radiation safety education 5. Sheyn DD, Racadio JM, Ying J, Patel MN, Racadio JM, Johnson ND. Efficacy of a radiation safety education
initiative in reducing radiation exposure in the pediatric IR suite. Pediatr Radiol 2008;38(6):669-674. https://doi initiative in reducing radiation exp
org $/ 10.1007 /$ s00247-008-0826-9

org/10.1007/s00247-008-0826-9
6. Rehani MM. Training of interventional cardiologists in radiation protection - the IAEA's initiatives. Int J Cardio 6. Rehani MM. Training of interventional cardiologists in radiation p
2007;114(2):256-260. https://doi.org/10.1016/.ijcard.2005.11.061

7. Fridell K, Ekberg J. Making the invisible visible: A qualitative study of the values, attitudes and norms of radiologists relating to radiation safety. J Radiol Protect 2016;36(2):200-214. https://doi.org/10.1088/0952 $4746 / 36 / 2 / 200$

8. Cole P, Hallard R, Broughton J, et al. Developing the radiation protection safety culture in the UK. J Radio Protect 2014;34(2):469-484. https://doi.org/10.1088/0952-4746/34/2/469

9. Sadigh G, Khan R, Kassin MT, Applegate KE. Radiation safety knowledge and perceptions among residents A potential improvement opportunity for graduate medical education in the United States. Acad Radio 2014;21(7):869-878. https://doi.org/10.1016/j.acra.2014.01.016

10. Rose A, Rae W, Chikobvu P, Marais W. A multiple methods approach: Radiation associated cataracts and occupational radiation safety practices in interventionalists in South Africa J Radiol Protect 2017:2(37):329-339. https://doi.org/ 10.1088/1361-6498/aaseee

11. Cousins C, Sharp C. Medical interventional procedures - reducing the radiation risks. Clin Radiol 2004;59(6):468473. https://doi.org/10.1016/j.crad.2003.11.014

12. Colleges of Medicine of South Africa. https://www.cmsa.co.za/default.aspx (accessed 13 October 2017).

12. Colleges of Medicine of South Africa. https://www.cmsa.co.za/default.aspx (accessed 13 October 2017).
13. Rose A, Rae WID. Perceptions of radiation safety training among interventionalists in South Africa. Cardiovasc Rose A, Rae WID. Perceptions of radiation safety training among
J Afr 2017;28(3):196-200. https://doi.org/10.5830/CVJA-2017-028

14. Kuon $\mathrm{E}$, Weitmann $\mathrm{K}$, Hoffmann $\mathrm{W}$, et al. Multicenter long-term validation of a minicourse in radiation-reducing techniques in the catheterization laboratory. Am J Cardiol 2015;115(3):367-373. https://doi.org/10.1016/. amjcard.2014.10.043

15. Azpiri-López JR, Assad-Morell JL, González-González JG, et al. Effect of physician training on the X-ray dose delivered during coronary angioplasty. J Invasive Cardiol 2013;25(3):109-113.

16. Georges J, Livarek B, Gibault-Genty G, et al. Reduction of radiation delivered to patients undergoing invasive coronary procedures. Effect of a programme for dose reduction based on radiation-protection training. Arch Cardiovasc Dis 2009;102(12):821-827. https://doi.org/10.1016/j.acvd.2009.09.007

17. Rose A, Uebel K, Rae W. Interventionalist perception on a culture of radiation protection. S Afr J Rad 2018;22(1):a1285. https://doi.org/10.4102/saj.v22i1.1285
} 